\title{
Über Zusammenhänge der Overhauser-Kernpolarisation mit der Molekülbewegung, der Frequenz und der Relaxation in Flüssigkeiten
}

\author{
Von K. D. Kramer und W. Müller-Warmuth \\ Max-Planck-Institut für Chemie (Otto-Hahn-Institut), Mainz \\ (Z. Naturforschg. 19 a, 375-387 [1964] ; eingegangen am 18. Dezember 1963)
}

\begin{abstract}
Die dynamische Kernpolarisation hängt in Flüssigkeiten, die gleichzeitig Kern- und Elektronenspins enthalten, von der Relativbewegung der die Wechselwirkungspartner tragenden Moleküle ab. Eine quantitative Untersuchung des Overhauser-Effektes gestattet daher Schlüsse auf die die Flüssigkeitsbewegung beschreibenden Größen. In drei organische Flüssigkeiten mit unterschiedlichem Viskositätsverhalten wurden zu diesem Zweck freie Radikalionen in Lösung gegeben und die dynamische (Lösungsmittel-) Protonenpolarisation im Temperatur- und Frequenzbereich zwischen den Grenzfällen des festen Zustandes und einer sehr schnellen Molekularbewegung gemessen. Der Abfall des Kern-Elektronen-Kopplungsparameters ist in den Radikallösungen mit einem rein translatorischen Diffusionsmechanismus verträglich und läßt sich quantitativ durch ein spezielles Flüssigkeitsmodell beschreiben. Als Einzelergebnisse werden u. a. der Temperaturgang der Korrelationszeiten und die "Grenzfrequenz“ des Overhauser-Effektes angegeben. Die gleichen Bewegungsmechanismen erklären die gemessenen Abhängigkeiten der Protonen- und der Elektronenspinrelaxationszeiten in den Lösungen freier Radikale. Die Interpretation erlaubt Aussagen über die Güte von Absolutberechnungen der Relaxationszeiten in Zwei-Spin-Systemen.
\end{abstract}

Untersuchungen des Overhauser-Effektes in Flüssigkeiten dienten bisher vor allem dem Nachweis einer vergrößerten Kernpolarisation überhaupt ${ }^{1-3}$, der „Verstärkung“ von magnetischen Kernresonanzsignalen ${ }^{4}$, der Realisierung eines „ProtonenMASERS" 1, 5, 6 und dem Studium der Elektronenresonanz-Linienstruktur und Relaxation ${ }^{7-12}$. Die vorliegende Arbeit bringt den Effekt in Zusammenhang mit der Flüssigkeitsstruktur. Da die Größe der dynamischen Kernpolarisation in Flüssigkeiten von der Art und der Zeitabhängigkeit der Kopplung zwischen Kern- und Elektronenspins abhängt, besteht eine enge Wechselbeziehung mit der Brownschen Molekularbewegung. Damit zusammenhängend gehen die benutzten Kern- und Elektronenresonanzfrequenzen in die Ergebnisse ein. Während bei den bisherigen Untersuchungen gewöhnlich angenommen wurde, daß alle Bewegungen in der Flüssigkeit schnell gegenüber der Periodendauer der eingestrahlten Hochfrequenzfelder ablaufen, stehen hier die $\mathrm{Zu}$ sammenhänge zwischen diesen Größen zur Diskus-

1 A. Abragam, J. Combrisson u. I. Solomon, C. R. Acad. Sci., Paris 245, 157 [1957].

2 L. H. Bennet u. H. C. Torrey, Phys. Rev. 108, 499 [1957].

3 A. Landesman, C. R. Acad. Sci., Paris 246, 1538 [1958].

${ }^{4}$ D. J. Parker, G. A. McLaren u. J. J. Conradi, J. Chem. Phys. 33, 629 [1960].

5 E. Allais, C. R. Acad. Sci., Paris 246, 2123 [1958].

6 M. Bonnet u. P. Servoz-Gavin, Bulletin Ampère 9 (Numéro spéc.), 629 [1960]. sion. Der Overhauser-Effekt nimmt mit steigender Zähflüssigkeit ebenso ab wie mit wachsenden Resonanzfrequenzen. Aus einem möglichst genauen quantitativen Studium des Polarisationseffektes erwartet man daher Aussagen über die Bewegungsmechanismen der die Spins tragenden Moleküle und über die für alle Anwendungen des Overhauser-Effektes wichtige „Frequenzgrenze“.

Für die Untersuchungen des Overhauser-Effektes kommen Flüssigkeiten in Frage, die zu verschiedenen Molekülen gehörende Kern- unid Elektronenspins enthalten. Da in diesen die für das Zustandekommen einer dynamischen Kernpolarisation wirksamen Mechanismen auch wesentlich zur Relaxation beitragen, liegt in Ergänzung eine Messung und Diskussion der Kern- und der Elektronenspinrelaxation auf der Hand. Wegen der größten Nachweisempfindlichkeit und des häufigen Vorkommens fanden als Kernspins ausschließlich Protonen Verwendung. In protonenhaltige Lösungsmittel, deren Zähigkeit sich mit der Temperatur in möglichst weiten Grenzen ändern läßt,

7 R. S. Codrington u. N. Bloembergen, J. Chem. Phys. 29, 600 [1958].

8 A. Landesman, J. Phys. Radium 20, 937 [1959].

9 E. H. Poindexter, J. Chem. Phys. 31, 1477 [1959].

10 W. Mülleer-W

11 J. Haupt, K. D. Kramer u. W. Müller-Warmuth, Magnetic and Electric Resonance and Relaxation, North-Holland Publ. Comp., Amsterdam 1962, S. 714.

12 J. Haupt u. W. Müller-W 1011 [1962]. 
wurden als Elektronenspins freie Radikalionen eingebaut. Deren magnetische Resonanz läßt sich nämlich verhältnismäßig leicht sättigen. Die Bereitstellung einer größeren Zahl von Meßfrequenzen stößt bei der komplizierten Doppelresonanztechnik auf gewisse Schwierigkeiten. Aus diesem Grunde lag eine Beschränkung auf mindestens zwei Frequenzen nahe, die aber möglichst weiten Abstand voneinander besitzen. Infolge der Abnahme des Overhauser-Effektes nach hohen Frequenzen hin lagen die höchsten Untersuchungsfrequenzen in einem Magnetfeld von etwa $1000 \mathrm{Gau} 3$ (Elektronenresonanz $3 \mathrm{GHz}$, Protonenresonanz 4,5 MHz). Die außerdem benutzten niedrigen Frequenzen von $43,5 \mathrm{MHz}$ und $65 \mathrm{kHz}$ in einem Magnetfeld von etwa $15 \mathrm{Gau} \Omega$ besitzen den Vorteil, bei genügend hohen Temperaturen den Grenzfall maximalen Overhauser-Effektes realisieren zu helfen.

Die dynamische Kernpolarisation kommt in dem Zweispin-System dadurch zustande, daß durch die möglichen strahlungslosen Übergänge zwischen den Zuständen des kombinierten Systems beim Induzieren von Elektronenresonanzübergängen die Protonenspins der Lösungsmittelmoleküle stärker ausgerichtet werden als normalerweise im gleichen Magnetfeld und bei der gleichen Temperatur. In Abschnitt 1 werden diese Zusammenhänge hergeleitet und insbesondere die Beziehungen zu naheliegenden Flüssigkeitsmodellen hergestellt. Den Aufbau der Anordnungen zur quantitativen Bestimmung des Vergrößerungsfaktors der dynamischen Kernpolarisation in Abhängigkeit der verschiedenen Parameter und zur Messung der Protonen- und Elektronenrelaxationszeiten beschreibt Abschnitt 2. In Abschnitt 3 werden für drei verschiedene Flüssigkeiten Ergebnisse von Messungen des Kern-ElektronenKopplungsparameters in Abhängigkeit von Temperatur und Frequenz sowie über die Korrelationszeiten mitgeteilt und die Konsequenzen für die oben gegebene Problemstellung diskutiert. Abschnitt 4 enthält Untersuchungen des durch Wechselwirkung mit den Elektronen erzeugten Protonenrelaxationsanteils und über den dipolaren Beitrag der Elektronenspinrelaxation.

\section{Der Overhauser-Effekt in einer Lösung mit Protonen- und Elektronenspins. Spezielle Flüssigkeitsmodelle}

Zur Beschreibung des Gesamtsystems wird von einer effektiven Spin-Hamilton-Funktion ausgegan- gen, die sich aus den Zeeman-Termen der Protonen(gyromagnetisches Verhältnis $\gamma_{I}$ ) und Elektronenspins $\left(\gamma_{S}\right)$, den zeitabhängigen Gliedern $\mathbf{H}_{S I}(t)$, $\mathbf{H}_{S S}(t)$ und $\mathbf{H}_{I I}(t)$ für Wechselwirkungen zwischen verschieden- und gleichartigen Spins und den Termen $\mathbf{H}_{S}^{\prime}(t)$ und $\mathbf{H}_{I}^{\prime}(t)$ für die Spin-Gitter-Wechselwirkungen (soweit diese nicht bereits in einem der anderen Operatoren enthalten sind) zusammensetzt $\mathbf{H}=\mathbf{H}_{\mathrm{o}}+\mathbf{H}^{\prime}=\gamma_{S} \hbar \mathbf{S} \boldsymbol{H}-\gamma_{I} \hbar \mathbf{I} \boldsymbol{H}+\mathbf{H}_{S I}(t)+$ $+\mathbf{H}_{S S}(t)+\mathbf{H}_{I I}(t)+\mathbf{H}_{S}^{\prime}(t)+\mathbf{H}_{I}^{\prime}(t)$.

Dabei ist angenommen worden, daß die Wechselwirkungsenergien mit dem äußeren Feld $\boldsymbol{H}$ groß gegenüber allen anderen Wechselwirkungen sind. Unter $\mathbf{H}_{S I}$ werden hier nur Wechselwirkungen zwischen Elektronen und Protonen betrachtet, die zu verschiedenen Molekülen gehören. Deren Abstandsvektoren sind durch die Brownsche Molekularbewegung unregelmäßig schwankende Funktionen der Zeit. Mögliche Wechselwirkungen der Elektronen mit moleküleigenen Kernmomenten werden bei der Diskussion des Sättigungsverhaltens der Elektronenresonanz getrennt berücksichtigt.

Dem ungestörten Намilton-Operator $\mathbf{H}_{0}$ entsprechen in einem magnetischen Gleichfeld $H_{z}=H_{0}$ für $S=I=1 / 2$ das Niveauschema von Abb. 1 und die vier Eigenzustände $\left.\mid m_{S}, m_{I}\right)$. Zwischen diesen las-

$$
\begin{aligned}
& \underline{E_{+}=1 / 2 \gamma_{s} \hbar H_{0} \quad N_{+}} m_{s}=+1 / 2 \\
& e_{-}=1 / 2 \gamma_{1} \hbar H_{0} \quad n_{-} m_{1}=-1 / 2 \\
& e_{+}=-1 / 2 \gamma_{1} \hbar H_{0} \quad n_{+} m_{I}=+1 / 2 \\
& \underline{E_{-}}=-1 / 2 \gamma_{S} \hbar H_{0} \quad N=m_{s}=-1 / 2
\end{aligned}
$$

Abb. 1. Energieniveauschema für ein statisch nicht gekoppeltes Elektronen-Protonen-Spinsystem.

sen sich durch geeignete Wechselfelder magnetische Dipolübergänge induzieren (Elektronen- und Protonenresonanz). Der Störoperator $\mathbf{H}^{\prime}(t)$ bewirkt strahlungslose Übergänge zwischen den Zuständen $k$ und $m$ (Relaxation), deren Wahrscheinlichkeit je Zeiteinheit man als Ergebnis einer zeitabhängigen Störungsrechnung 1 . Ordnung erhält

$$
\begin{gathered}
\overline{w_{k m}}=\frac{1}{\hbar^{2}} \int_{0}^{t}\left(k\left|\mathbf{H}^{\prime}(t)\right| m\right)\left(m\left|\mathbf{H}^{\prime}\left(t^{\prime}\right)\right| k\right) \\
\quad \cdot \exp \left\{i \omega_{k m}\left(t-t^{\prime}\right)\right\} \mathrm{d} t^{\prime}+\text { konj. kompl. } \\
\text { mit } \quad \hbar \omega_{k m}=(E+e)_{m}-(E+e)_{k} .
\end{gathered}
$$

Wegen des statistischen Charakters von $\mathbf{H}^{\prime}(t)$ kann als Meßgröße nur ein zeitlicher Mittelwert angege- 
ben werden. Ein Protonenspin kann sich im allgemeinen Fall vermittels $\mathbf{H}^{\prime}(t)$ von $m_{I}=+1 / 2$ nach $m_{I}=-1 / 2$ (bzw. umgekehrt) umorientieren, wenn gleichzeitig ein Elektronenspin von $m_{S}=-1 / 2$ nach $m_{S}=+1 / 2$ (bzw. umgekehrt) umklappt (Übergangswahrscheinlichkeit $w_{0}$ ), wenn ein Elektronenspin von $+1 / 2$ in $-1 / 2$ übergeht $\left(w_{2}\right)$ oder seinen $\mathrm{Zu}$ stand überhaupt nicht ändert $\left(w_{1}\right)$. Die $w_{i}$ ergeben sich aus der Mittelung von Schwankungsgrößen nach (2) und der speziellen Form von $\mathbf{H}^{\prime}(t)$.

Durch die induzierten und strahlungslosen Übergänge kann die Verteilung der $N=N_{+}+N_{-}$Elektronenspins und der $n=n_{+}+n_{-}$Kernspins auf die Energieniveaus von Abb. 1 geändert werden. Für die Änderungsrate der Besetzungszahlen durch Relaxation können, ähnlich wie zuerst von SoLomon durchgeführt ${ }^{13}$, makroskopische Bewegungsgleichungen aufgestellt werden:

$$
\begin{aligned}
\frac{\mathrm{d} n_{+}}{\mathrm{d} t}=-\frac{\mathrm{d} n_{-}}{\mathrm{d} t}=-n_{+} & {\left[w_{0} \frac{N_{-}}{N} e^{-\left(\Pi_{0}+P_{0}\right)}\right.} \\
& \left.+w_{2} \frac{N_{+}}{N} e^{\left(\Pi_{0}-P_{0}\right)}+w_{1} e^{-P_{0}}\right] \\
+ & n_{-}\left[w_{0} \frac{N_{+}}{N} e^{\left(\Pi_{0}+P_{0}\right)}\right. \\
& \left.+w_{2} \frac{N_{-}}{N} e^{-\left(\Pi_{0}-P_{0}\right)}+w_{1} e^{P_{0}}\right] .
\end{aligned}
$$

Da alle Übergänge strahlungslos durch Kopplung an ein „Wärmebad“ der Temperatur $T$ zustande kommen, müssen die mittleren Übergangswahrscheinlichkeiten $w_{i}$ in (3) mit dem zugehörigen BoltzmanNFaktor multipliziert werden $\left(\Pi_{0}=\gamma_{S} \hbar H_{0} / 2 k T\right.$, $\left.P_{0}=\gamma_{I} \hbar H_{0} / 2 k T\right)$ ). Für die Übergänge, an denen auch Elektronenspins beteiligt sind, geht multiplikativ die Wahrscheinlichkeit ein, daß das Elektron sich in dem betreffenden Zustand befindet. Geht man auf die Besetzungszahldifferenz über und definiert als Elektronen- bzw. Protonenpolarisation in Feldrichtung

$$
\Pi=\frac{N_{-}-N_{+}}{N} \quad \text { und } \quad P=\frac{n_{+}-n_{-}}{n},
$$

so ergibt (3), wenn man eine Potenzreihenentwicklung der Exponentialfunktionen wegen der Kleinheit der Exponenten nach dem linearen Gliede abbricht

$$
\begin{aligned}
\frac{\mathrm{d} P}{\mathrm{~d} t}= & -\left(w_{0}+2 w_{1}+w_{2}\right) \\
\cdot & {\left[\left(P-P_{0}\right)-\frac{w_{2}-w_{0}}{w_{0}+2 w_{1}+w_{2}}\left(\Pi-\Pi_{0}\right)\right], }
\end{aligned}
$$

$w_{0}$ und $w_{2}$ werden durch Kern-Elektronenspin-Wechselwirkungen $\mathbf{H}_{S I}$ erzeugt, $w_{1}=w_{1}{ }^{\prime}+w_{1}{ }^{\prime \prime}$ ebenfalls durch diese $\left(w_{1}^{\prime}\right)$, aber auch vermittels $\mathbf{H}_{I I}$ und $\mathbf{H}_{I}^{\prime}\left(w_{1}^{\prime \prime}\right)$. (4 a) schreibt sich deshalb vorteilhafter

$$
\frac{\mathrm{d} P}{\mathrm{~d} t}=-\frac{1}{T_{1}}\left[\left(P-P_{0}\right)-Q f\left(\Pi-\Pi_{0}\right)\right]
$$

mit

$$
\varrho=\frac{w_{2}-w_{0}}{w_{0}+2 w_{1}^{\prime}+w_{2}}
$$

(Kern-Elektronen-Kopplungsparameter),

$$
f=\frac{w_{0}+2 w_{1}^{\prime}+w_{2}}{w_{0}+2\left(w_{1}^{\prime}+w_{1}^{\prime \prime}\right)+w_{2}}
$$

(Streufaktor für den Bruchteil der durch Wechselwirkung mit den Elektronen erzeugten Kernrelaxation), und $\frac{1}{T_{1}}=w_{0}+2\left(w_{1}{ }^{\prime}+w_{1}^{\prime \prime}\right)+w_{2}$.

Wenn das Elektronenspinsystem vermöge $\mathbf{H}_{S S}$ und $\mathbf{H}_{S}^{\prime}$ einen eigenen viel stärkeren Relaxationsmechanismus besitzt, ist $T_{1}$ die Zeitkonstante, mit der das Kernspinsystem nach einer Störung in das Gleichgewicht übergeht. Im stationären Zustand ist $\mathrm{d} P / \mathrm{d} t$ gleich Null, und der Vergrößerungsfaktor der dynamischen Kernpolarisation beträgt

mit

$$
\begin{gathered}
\frac{P}{P_{0}}=1-o f s \frac{\gamma_{S}}{\gamma_{I}}=1-F s \\
s=\left(\Pi_{0}-\Pi\right) / \Pi_{\mathbf{0}} .
\end{gathered}
$$

Durch Induzierung von Elektronenresonanzübergängen $\Delta m_{S}=1$ läßt sich für die Elektronenspins eine Ungleichgewichtsverteilung $(0<s \leqq 1)$ herstellen und unter Umständen eine gegenüber dem Gleichgewichtswert $P_{0}$ beträchtlich vergrößerte Kernpolarisation $P$ erzeugen (Overhauser-Effekt) . (5) gilt für beliebige $I$ und $S$. In einer früheren Arbeit ${ }^{12}$ wurden die Zusammenhänge zwischen dem Sättigungsparameter $s$ und den Eigenschaften der Elektronenresonanz unter besonderer Berücksichtigung einer Hyperfeinstruktur hergeleitet. Diese Ergebnisse werden benutzt, um das Hauptziel der vorliegenden Untersuchung, eine Messung des Kopplungsparameters $\varrho$ in Abhängigkeit der Frequenz und der Flüssigkeitseigenschaften, zu erreichen.

Zur Berechnung von $\varrho$ wird von einem magnetischen Wechselwirkungsoperator ausgegangen, der sich aus einem Dipol-Dipol- und einem Fermi-Kontakt-Anteil zusammensetzt

$$
\mathbf{H}_{S I}(t)=-\frac{\gamma_{S} \gamma_{I} \hbar^{2}}{r(t)^{3}}\left[\mathbf{S I}-3 \frac{(\mathbf{S} \boldsymbol{r}(t))(\mathbf{I} \boldsymbol{r}(t))}{r(t)^{2}}\right]+A(t) \mathbf{S I} .
$$

In Koordinationsschreibweise geht $(6$ a) in eine Summe über, deren Glieder Produkte aus einem

${ }^{13}$ I. Solomox, Phys. Rev. 99, 559 [1955]. 
zeitunabhängigen Operatoranteil $\mathfrak{U}_{j}$ und einer statistisch schwankenden (klassischen) Funktion $F_{j}(t)$ sind,

$$
\mathbf{H}_{S I}(t)=\sum_{j} \mathfrak{U}_{j} F_{j}(t) .
$$

Die explizite Form dieser Umrechnung ist in der Literatur gegeben ${ }^{14}$. Unter Berücksichtigung von (6) und Einführung der Autokorrelationsfunktion der Schwankungsfunktion $F_{j}(t)$ bezüglich eines Zeitintervalles $\tau=t^{\prime}-t$,

$$
K_{j}(\tau)=\overline{F_{j}(t) \cdot F_{j}^{*}(t+\tau),}
$$

ergibt (2) für den Störanteil $\mathbf{H}_{S I}$

$$
\begin{gathered}
\overline{w_{-+}}=\frac{1}{2} w_{0}+w_{1}{ }^{\prime}+\frac{1}{2} w_{2} \\
=\frac{1}{\hbar^{2}} \sum_{j}\left|\left(+\left|\mathfrak{U}_{j}\right|-\right)\right| 2 \int_{-\infty}^{\infty} K_{j}(\tau) \exp \left\{-i \omega_{j} \tau\right\} \mathrm{d} \tau .
\end{gathered}
$$

Dabei ist bereits vorausgesetzt, da $\beta$ es sich bei der BRowsschen Molekularbewegung um einen stationären Schwankungsprozeß handelt, und da $ß$ die Korrelationsfunktion sehr viel schneller abklingt als die betrachteten Zeiten $t$. Als Flüssigkeitseigenschaft enthält (8) die Spektraldichtefunktionen

$$
J_{j}\left(\omega_{j}\right)=\int_{-\infty}^{\infty} K_{j}(\tau) \exp \left\{-i \omega_{j} \tau\right\} \mathrm{d} \tau
$$

und

$$
\begin{aligned}
& J_{A}\left(\omega_{S}+\omega_{I}\right)=\int_{-\infty}^{\infty} \overline{A(t) \cdot A^{*}(t+\tau)} \\
& \cdot \exp \left\{-i\left(\omega_{S}+\omega_{I}\right) \tau\right\} \mathrm{d} \tau .
\end{aligned}
$$

Mit den speziellen Werten für die Matrixelemente von $\mathfrak{U}_{j}$ liefert (8)

$$
\begin{aligned}
w_{0} & =\frac{1}{16} \gamma_{S}^{2} \gamma_{I}^{2} \hbar^{2} J_{0}\left(\omega_{S}+\omega_{I}\right)+\frac{1}{4 \hbar^{2}} J_{A}\left(\omega_{S}+\omega_{I}\right), \\
w_{1}{ }^{\prime} & =\frac{9}{16} \gamma_{S}^{2} \gamma_{I}^{2} \hbar^{2} J_{1}\left(\omega_{I}\right), \\
w_{2} & =\frac{9}{16} \gamma_{S}^{2} \gamma_{I}^{2} \hbar^{2} J_{2}\left(\omega_{S}-\omega_{I}\right) .
\end{aligned}
$$

Für den Kopplungsparameter $\varrho$ ergibt sich damit für reine Dipol-Dipol-Wechselwirkung $\left(J_{A}=0\right)$ :

$$
\varrho=\frac{9 J_{2}\left(\omega_{S}-\omega_{I}\right)-J_{0}\left(\omega_{S}+\omega_{I}\right)}{J_{0}\left(\omega_{S}+\omega_{I}\right)+18 J_{1}\left(\omega_{I}\right)+9 J_{2}\left(\omega_{S}-\omega_{I}\right)},
$$

für reine Austauschwechselwirkung: $\varrho=-1$.

Um weitere Aussagen über die Funktionen $J_{j}(\omega)$ zu gewinnen, muß ein spezielles Modell für die Flüssigkeitsbewegung gewählt werden. Für das $\mathrm{Zu}$ -

14 A. Abragam, Nuclear Magnetism, Clarendon Press, Oxford 1961, S. 289 u. 308. standekommen der Übergangswahrscheinlichkeiten (10) und der Kern-Elektronen-Kopplung (11) sind die Bewegungen der die Spins tragenden Moleküle oder Molekülkomplexe maßgeblich. Dabei genügt für das vorliegende Problem die Kenntnis des FouRIER-Spektrums der sich statistisch verändernden Bewegungskoordinaten. Da der in den Funktionen $F_{j}$ auftretende Abstandsvektor $\boldsymbol{r}$ zwischen zwei Wechselwirkungspartnern eine statistische Variable ist, sind nur Wahrscheinlichkeitsaussagen möglich, welche aber die Mittelwertsbildungen ( $7 \mathrm{a})$ gestatten. Wenn $P\left(\boldsymbol{r}_{0}, \boldsymbol{r}, \tau\right)$ die Wahrscheinlichkeit bedeutet, zu Anfang und Ende eines Zeitintervalls $\tau$ die Verbindungsvektoren $\boldsymbol{r}_{0}$ und $\boldsymbol{r}$ vorzufinden, läßt sich die zeitliche Mittelung ( $7 \mathrm{a}$ ) durch eine räumliche ausdrücken,

$K(\tau)=\iint P\left(\boldsymbol{r}_{0}, \boldsymbol{r}, \tau\right) \cdot F_{j}\left(\boldsymbol{r}_{0}\right) F_{j}^{*}(\boldsymbol{r}) \mathrm{d} \boldsymbol{r}_{0} \mathrm{~d} \boldsymbol{r}$.

Die Integration ist darin über die erlaubten Wertebereiche des Koordinatenraumes zu erstrecken. Bei Benutzung von Kugelkoordinaten durchlaufen $\vartheta$ und $\varphi$ alle Werte, während $r$ sein Minimum in $d$ besitzt, dem kleinstmöglichen Abstand der Partner (wenn die Moleküle zusammenstoßen). Als ein den quantitativen Vergleich mit Meßdaten ermöglichendes Modell soll an dieser Stelle angenommen werden, daß die Bewegungen aller beteiligten Moleküle der „Diffusionsgleichung“ genügen, einem Ausgleichsgesetz für irreversible Vorgänge, wie es bei ähnlichen Problemen in der Physik häufig auftritt. Das heißt, für die Wahrscheinlichkeitsdichte $P\left(\boldsymbol{r}_{0}, \boldsymbol{r}, \tau\right)$ soll gelten

$$
\partial P / \partial t=D \cdot \Delta P
$$

Die Vielfalt der statistischen Bewegungsvorgänge macht eine Unterscheidung zwischen mehreren Einzelbewegungstypen sinnvoll. Wenn einmal von Austauschwechselwirkungen abgesehen wird, sind das die Relativbewegungen zwischen verschiedenen Molekülen und die rotatorischen Taumelbewegungen ganzer Komplexe. Während die erste Bewegungsart von vornherein naheliegt, da die wechselwirkenden Spins zu verschiedenen Molekülen gehören, weiß man aus Untersuchungen der Protonenrelaxation wäßriger Lösungen von paramagnetischen Ionen, daß der Hauptbeitrag gerade von Kern-ElektronenWechselwirkungen der in der Hydrathülle des Ions befindlichen Wassermoleküle herrührt. Im Falle der Relativbewegung zweier diffundierender Moleküle (Diffusionskonstanten $D_{1}$ und $D_{2}$ ) führt (12) unter 
den gegebenen Anfangsbedingungen zu der Lösung ${ }^{15}$

$$
P\left(\boldsymbol{r}_{0}, \boldsymbol{r}, \tau\right)=\frac{\exp \left\{-\left(\boldsymbol{r}-\boldsymbol{r}_{0}\right)^{2} / 4\left(D_{1}+D_{2}\right) \tau\right\}}{\sqrt{4 \pi\left(D_{1}+D_{2}\right)^{3}}} .
$$

Die aus der Diffusion zweier Teilchen gewonnenen Ergebnisse lassen sich auf die Gesamtheit von $N$ Spins übertragen, wenn deren Bewegungen nicht korreliert sind. (7 b) ist dann mit $N$ zu multiplizieren. Zur Berechnung von $K(\tau)$ werden (13) und die speziellen Werte von $F_{j}(\boldsymbol{r})$ in $(7 \mathrm{~b})$ eingesetzt und die Integrationen durchgeführt. Diese etwas langwierige Rechnung ist in der Literatur für den Fall durchgeführt worden, daß in den Funktionen $F_{j}(\boldsymbol{r})$ die Abstandsschwankungen $r(t)$ groß gegenüber den Winkelschwankungen $\vartheta(t)$ und $\varphi(t)$ sind, also für die Translationsbewegung ${ }^{16}$. Es ergibt sich dann eine Korrelationsfunktion

$$
\begin{aligned}
K_{j}(\tau)=c_{j} & \frac{N}{d^{3}} \int_{0}^{\infty} I_{3 / 2}^{2}(u) \\
& \cdot \exp \left\{-\left(D_{1}+D_{2}\right)|\tau| u^{2} / d^{2}\right\} \frac{\mathrm{d} u}{u},
\end{aligned}
$$

$I_{3 / 2}(u)=$ BesseL-Funktion der Ordnung $3 / 2$,

$$
c_{0}=\frac{48}{15} \pi, \quad c_{1}=\frac{8}{15} \pi \quad \text { und } \quad c_{2}=\frac{32}{15} \pi .
$$

Mit der Definition $\quad \tau_{\mathrm{c}}=\frac{d^{2}}{3\left(D_{1}+D_{2}\right)}$

für die Korrelationszeit der translatorischen Bewegung führt die Fourier-Transformation (9) zu einer Spektraldichtefunktion

$$
J_{j}(\omega)=6 c_{j} N \frac{\tau_{\mathrm{c}}}{d^{3}} \int_{0}^{\infty} I_{3 / 2}^{2}(u) \frac{u}{u^{4}+9 \omega^{2} \tau_{\mathrm{c}}{ }^{2}} \mathrm{~d} u .
$$

Das Integral in (16) wurde mittels der Substitutionen

$I_{3 / 2}^{2}(u)=\sqrt{\frac{2}{\pi}} \frac{1}{1 / u}\left(\frac{1}{u} \sin u-\cos u\right)$ und $\alpha=9 \omega^{2} \tau_{\mathrm{c}}{ }^{2}$

programmiert und maschinell auf einer IBM 7090 für die Parameterwerte $\alpha=9 \cdot 10^{n / 2}$ mit $-16 \leqq n$ $\leqq 8$ ausgewertet ${ }^{*}$. Der Verlauf ist in Abb. 2 wiedergegeben. Die für die Auswertung der experimentellen Ergebnisse wichtigen Funktionswerte für große $\omega \tau_{\mathrm{e}}$ sind vergrößert herausgezeichnet worden. Der Kopplungsparameter (11) läßt sich vermittels der

15 A. Sommerfeld, Partielle Differentialgleichungen der Physik, Dieterichsche Verlagsbuchh., Wiesbaden 1947, S. 55 ff.

16 P. S. Hurbard, Phys. Rev. 131, 275 [1963] und Anm. ${ }^{14}$, S. 301.

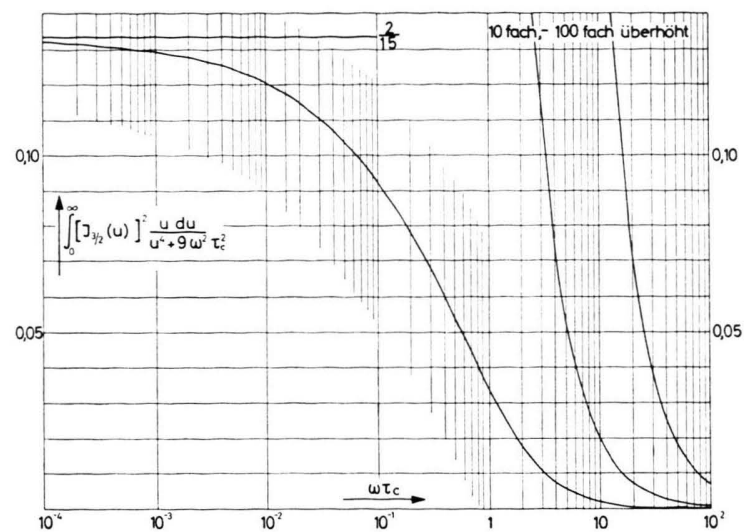

Abb. 2. Funktionaldarstellung des maschinell ausgewerteten, in der Spektraldichtefunktion der translatorischen Diffusion auftretenden Integrals.

in (14) gegebenen $c_{j}$ und wegen $\omega_{S} \gg \omega_{I}$ auf zwei Spektralfunktionen zurückführen

$$
\begin{array}{r}
\varrho=\frac{1}{2} \cdot \frac{1}{0,7+0,3 J\left(\omega_{I}\right) / J\left(\omega_{S}\right)} \\
{\left[J(\omega) \equiv J_{j}(\omega) / c_{j}\right] .}
\end{array}
$$

Für die rotatorischen Diffusionsbewegungen können ähnliche Überlegungen angestellt werden, nur daß der Abstand zwischen den Wechselwirkungspartnern eine Konstante ist. Das vereinfacht das Integral (7 b) mit der für diesen Fall gültigen Lösung von (12) beträchtlich ${ }^{17}$ und führt schließlich zu

$$
J_{j}^{\text {rot }}(\omega)=\frac{c_{j}}{4 \pi} \frac{1}{b^{6}} \frac{2 \tau_{\mathrm{c}}^{\text {rot }}}{1+\omega^{2} \tau_{\mathrm{c}}^{2, \text { rot }}} .
$$

Darin ist als rotatorische Korrelationszeit definiert

$$
\tau_{\mathrm{c}}^{\mathrm{rot}}=a^{2} / 6 D_{\mathrm{rot}} .
$$

$a$ bedeutet den effektiven Radius des diffundierenden Molekülkomplexes, $b$ den Abstand zwischen zwei wechselwirkenden Spins. An Stelle von (17) ergibt sich damit

$$
\varrho^{\mathrm{rot}}=\frac{1}{2} \frac{1}{0,7+0,3\left(1+\omega_{S}^{2} \tau_{\mathrm{c}}^{2, \mathrm{rot}}\right) /\left(1+\omega_{I}^{2} \tau_{\mathrm{c}}^{2, \text { rot }}\right)} .
$$

Für den Grenzfall $\omega_{S} \tau_{\mathrm{c}} \ll 1$ gehen (17) und (20) in $\varrho=1 / 2$ über.

\section{Experimentelles}

Die für die vorliegenden Untersuchungen benötigten Doppelresonanz-Meßanordnungen unterschieden sich

\footnotetext{
* Für die Durchführung dieser Integration sind wir Herrn Dipl.-Math. W. Thiele zu besonderem Dank verpflichtet. 17 Anm. ${ }^{14}$, S. 298.
} 
von den herkömmlichen Kern- und Elektronenresonanzspektrometern vor allem dadurch, daß zwei hochfrequente Wechselfelder erforderlich sind. Das eine bei der (höheren) Frequenz $\omega_{S}$ dient zur Anregung von Elektronenresonanzübergängen und muß über die zur weitgehenden Sättigung erforderliche Leistung verfügen, das andere (Frequenz $\omega_{I}$ ) wird zum Nachweis der dynamischen Polarisation durch Kernresonanz gebraucht. Das apparative Problem ist dabei, beide Hochfrequenzfelder so an die Probe heranzuführen, daß durch die gegenseitige Beeinflussung möglichst wenig Leistungs- und Empfindlichkeitsverlust entsteht. Neben der eigentlichen Messung des Overhauser-Effektes sollten die Spinkonzentrationen der Radikallösungen, die elektronischen Linienstrukturen und Relaxationszeiten und für den Streufaktor $f$ die Protonenrelaxationszeiten bestimmt werden können. Für alle diese Untersuchungen fanden Verwendung:

1. ein Mikrowellen-Doppelresonanzspektrometer mit $\omega_{S}=2 \pi \cdot 3,0 \mathrm{GHz}$ und $\omega_{l}=2 \pi \cdot 4,5 \mathrm{MHz}$, dessen Blockschaltbild unten gegeben ist, Magnetfeld $1070 \mathrm{Gauß,}$

2. ein Schwachfeld-Doppelresonanzspektrometer mit $\omega_{S}=2 \pi \cdot 20$ bis $2 \pi \cdot 90 \mathrm{MHz}$, dessen wesentliche Merkmale bereits in früheren Arbeiten beschrieben worden sind 10, 12, Helmholtz-Magnetfeld 15 Oersted,

3. ein $3 \mathrm{GHz}$-Elektronenresonanzspektrometer ${ }^{18}$,

4. eine Anordnung zur Messung von Kernrelaxationszeiten im schwachen Magnetfeld ${ }^{19}$ und

5. eine Impulsapparatur zur Messung von Kernrelaxationszeiten bei $4,5 \mathrm{MHz}$.

Das unter 1. genannte, für Messungen des Vergrößerungsfaktors der dynamischen Kernpolarisation aufgebaute Spektrometer ist in Abb. 3 im Blockschaltbild dargestellt. Zur Erzeugung der Mikrowellenleistung

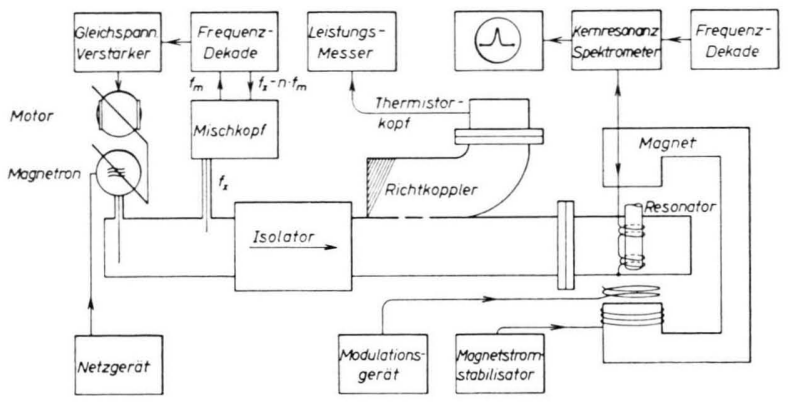

Abb. 3. Blockschema des Mikrowellen-Doppelresonanzspektrometers.

dient ein Dauerstrich-Hochleistungsmagnetron vom Typ $\mathrm{R}$ a y th e on QK 61. Es arbeitet auf einem $H_{102}$-Rechteckresonator als Last, der mittels auswechselbarer Blenden angepaßt wird. Der zur Verhinderung einer schädlichen Reflexion benötigte Hochleistungsisolator kann im Höchstfall $60 \mathrm{Watt}$ in Sperr-Richtung aufnehmen und wurde selbst gebaut. Sein Isolatoreffekt beruht auf

18 K. D. Kramer u. W. Müller-Warmuth, Z. Angew. Phys. 16, 281 [1963]. der Elektronenresonanzabsorption in Ferriten ${ }^{20}$. Die auf den Resonator treffende Leistung wurde durch eine kommerzielle Thermistor-Meßbrücke ermittelt, die über einen geeichten Richtkoppler den Vorlauf anzeigt. Da man für die Frequenzstabilisation des Magnetrons nicht auf eine Frequenz-Spannungsabhängigkeit zurückgreifen konnte, wurde hier die Differenz gegenüber einer Quarzfrequenz zur Regelung eines Servomotors benutzt, der auf die mechanische Abstimmung des Magnetrons einwirkt.

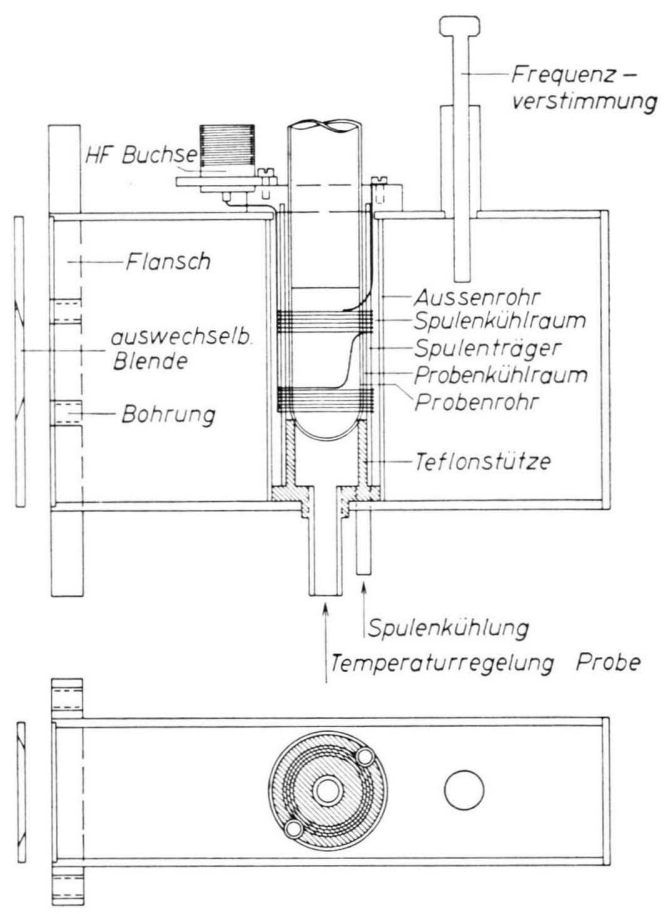

Abb. 4. Doppelresonanzresonator.

Im Innern des Resonators (Abb. 4) wird neben der ESR-Anregung auch der Kernresonanznachweis vorgenommen. Die Flüssigkeitsprobe befindet sich am Orte größten magnetischen und damit kleinsten elektrischen Mikrowellenfeldes. Die Kernresonanzspule als Bestandteil eines elektrischen Schwingkreises ist in zwei Paketen angeordnet, um der Mikrowelle Zutritt zur Probe zu gestatten. Dadurch sinkt zwar der KernresonanzFüllfaktor; es wird aber weitgehend verhindert, daß die Mikrowelle an den Drähten reflektiert wird und daß die Wicklungen den Charakter von Kurzschlußwindungen erhalten. Das Kernresonanzspektrometer für 4,5 MHz besitzt übliche Bauart: „Q-Meter", Verstärker, phasenempfindlicher Detektor, Schreiber. Als Besonderheiten sind nur die Vorkehrungen gegen eine geringfügige Erwärmung der Spule zu erwähnen, die bei Einstrahlung starker Mikrowellenleistungen trotz Kühlung

19 J. Haupt u. W. Müller-W [1962].

20 C. Kitrel, Phys. Rev. 73, 155 [1948]. 
noch eintritt und zu Frequenz- und Güteänderungen des Schwingkreises führt. Diese bestehen in der Verwendung einer stabilen Kernresonanzfrequenz (Quarzdekade) und in der Anwendung eines früher beschriebenen „schnellen“ Modulationsverfahrens ${ }^{21}$. Da hier nur die relative Intensität des Signals interessiert, um Vergrößerungsfaktoren der dynamischen Kernpolarisation zu messen, werden dabei die Parameter so eingestellt, daß der Arbeitspunkt in der flachen Mulde zwischen dicht beieinanderliegenden Zentral- und Seitenbändern der Dispersionslinie liegt. Schwankungen sind dann mit nur unmerklichen Signaländerungen verbunden.

Bei allen anfangs aufgeführten Messungen mußten für das vorliegende Problem die Probentemperaturen zwischen etwa $-70{ }^{\circ} \mathrm{C}$ und $+100^{\circ} \mathrm{C}$ geändert werden können. Die Proben von etwa $10 \mathrm{~cm}^{3}$ Volumen wurden dazu durch Einbringen in ein Wärmebad vortemperiert und dann in den Spektrometern durch einen Strom verdampfenden flüssigen Stickstoffs gekühlt bzw. durch Preßluft erhitzt, die ein heißes Ölbad durchströmt hatte. Die Einstellung der Probentemperatur geschah durch Regelung der Strömungsgeschwindigkeiten. In dem Resonator von Abb. 4 sind um das Probengefäß zwei Quarzglasrohre konzentrisch angeordnet. Im Inneren strömt die zur Temperaturegelung benutzte Luft. Der Raum zwischen beiden Glasrohren wird zusätzlich von getrockneter Luft durchströmt, um im Falle tiefer Kühlung das Ansetzen von Schnee zu verhindern.

Die Darstellung der radikalischen Lösungen von Diphenylradikalionen in Tetrahydrofuran (THF), Dimethylgykol (DMG) und Dimethyldiglykol = Diäthylenglykoldimethyläther (DGDE) erfolgte durch Reduktion von Diphenyl mit metallischem Natrium in den genannten Lösungsmitteln, die gute Elektronenübertragungseigenschaften besitzen. Die Lösungen müssen absolut frei von Wasser und Sauerstoff sein. Das wird durch zwölfstündige Rückflußdestillation unter ständigem Durchperlen von sauerstoffreiem Stickstoff erreicht. Die Reaktion erfolgt im überdestillierten Lösungsmittel unter Luftausschluß. Die Probengefäße wurden im ausgefrorenen Zustand abgeschmolzen. Die Umwandlungstemperaturen liegen bei den verwandten Lösungen wie folgt: THF, Festpunkt bei $-60{ }^{\circ} \mathrm{C}$, Kochpunkt bei $+65{ }^{\circ} \mathrm{C}$; DMG, FP bei $-75{ }^{\circ} \mathrm{C}$, KP bei $+85{ }^{\circ} \mathrm{C}$; DGDE, FP bei $-70{ }^{\circ} \mathrm{C}$, KP bei $+165^{\circ} \mathrm{C}$. Die zwischen diesen Fixpunkten durch Temperaturänderungen erzielbaren Unterschiede im Bewegungsspektrum wurden für die vorliegenden Untersuchungen ausgenutzt. Die Radikalkonzentration spielte hier nur eine untergeordnete Rolle. Aus Empfindlichkeitsgründen, damit der Faktor $f$ in $(4 \mathrm{~b})$ nicht zu klein wird, und um ein austauschverschmälertes Elektronenresonanzspektrum $\mathrm{zu}$ erhalten, wurde sie nicht zu klein gewählt.

Die Meßmethodik bestand darin, für die einzelnen Proben bei verschiedenen Temperaturen und verschiedenen Frequenzen aus Kernresonanzmessungen den Vergrößerungsfaktor der dynamischen Kernpolarisation

21 W. Müller-Warmuth u. P. Parikh, Z. Naturforschg. 16 a, 1063 [1961].
(5) zu bestimmen. Bei der Durchführung wurde zunächst mit dem Elektronenresonanzspektrometer das vollständige ESR-Spektrum aufgenommen, um Linienstruktur und Radikalkonzentration zu ermitteln. Für die jeweilige Meßtemperatur wurden dann die Resonanzfrequenzen der Protonen- und Elektronenspins im richtigen Verhältnis zueinander eingestellt und die „Sättigungskurven“ gemessen, ähnlich wie es früher ${ }^{12}$ von uns angegeben wurde, um auf $s=1$ zu extrapolieren. $f$ ergab sich durch getrennte Messung der Protonenrelaxation bei der betreffenden Frequenz und Temperatur in den Lösungen und in den reinen Lösungsmitteln.

\section{Ergebnisse und Diskussion}

Abb. 5 zeigt die Meßergebnisse für den VergröBerungsfaktor $P / P_{0}$ der dynamischen Protonenpolarisation für eine der Proben als Beispiel. Aufgetragen ist praktischerweise $1 /\left(P / P_{0}-1\right)$ als Funktion von $1 / N_{\text {Mikro }}$ (Mikrowellenleistung $N_{\text {Mikro }}$ ). Je nach

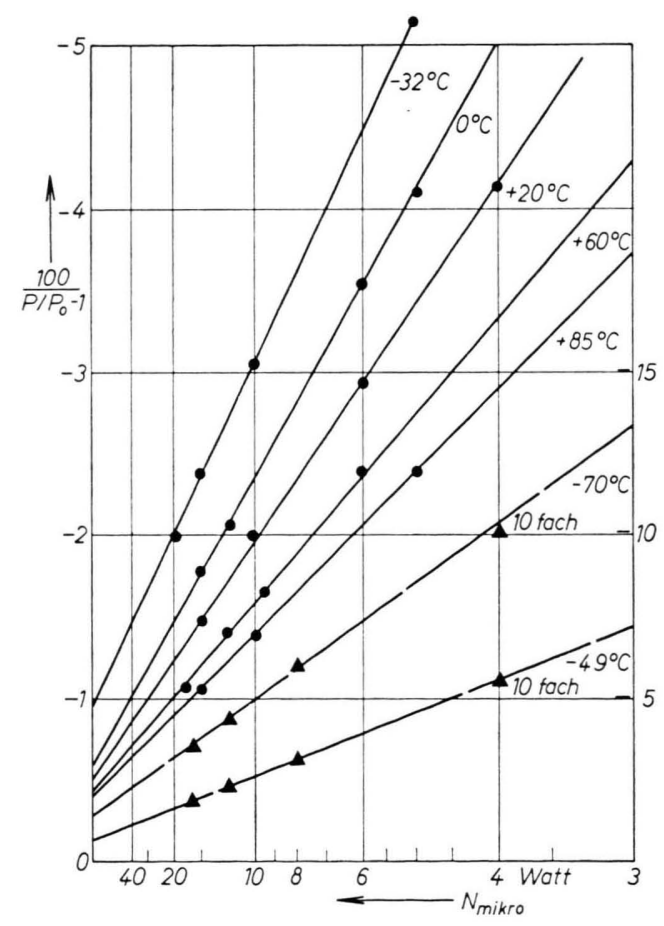

Abb. 5. „Sättigungskurven“ für eine DGDE-Lösung mit einer $2,9 \cdot 10^{-2}-m$. Diphenylradikalkonzentration.

den Eigenschaften des Elektronenresonanzspektrums ist diese Größe nämlich $1 / H_{1}{ }^{2}$ bzw. $1 / H_{1}$ oder einem Zwischenwert proportional ${ }^{12} \quad\left(H_{1}=\right.$ magnetische Mikrowellenfeldkomponente). Die lineare Abhängigkeit von der inversen Mikrowellenleistung in 
Abb. 5 weist darauf hin, daß das Sättigungsverhalten hier dem einer einzelnen ESR-Linie nahekommt. Tatsächlich haben Austauschwechselwirkungen zwischen den Elektronenspins das durch Hyperfeinkopplung des ungepaarten Elektrons an die moleküleigenen Protonen erzeugte Viellinienspektrum in eine Einzellinie zusammenfallen lassen und diese verschmälert. Das ist für die Gültigkeit von (5) nicht notwendig erforderlich. Der Ausdruck (5) und die benutzte Meßmethodik gilt - wie in Anm. ${ }^{12}$ im einzelnen gezeigt wurde - für beliebige Elektronenresonanzstrukturen. Bei kleineren Radikalkonzentrationen biegen die Kurven in einer der Abb. 5 entsprechenden Darstellung allerdings parabelförmig nach unten um. Aus den Schnittpunkten der Kurven von Abb. 5 mit der Ordinatenachse erhält man nach (5) den auf volle Sättigung $(s=1)$ extrapolierten Vergrößerungsfaktor $F$. Dieser wurde für alle Probenlösungen für beide Frequenzen bestimmt.

Für jeden Arbeitspunkt wurden außerdem unter den gleichen Bedingungen die Protonenrelaxationszeiten in den reinen Lösungsmitteln $\left(T_{10}\right)$ und in den Probenlösungen $\left(T_{1}\right)$ gemessen, um den in $(4 \mathrm{~b})$ definierten Streufaktor $f=1-T_{1} / T_{10}$ zu ermitteln. Als Ergebnis lassen sich nach (5) Werte für $\varrho \cdot \gamma_{S} / \gamma_{I}$ gewinnen. Wie die Messungen im einzelnen zeigen, streben diese in allen Fällen für kleine Frequenzen und hohe Temperaturen dem Grenzwert $330 \mathrm{zu}$, einem Vergrößerungsfaktor, der bei der Meßfrequenz von $43 \mathrm{MHz}$ wirklich erreicht wurde. Daraus folgt nach den Ausführungen von Abschnitt 1: $\varrho$ ist gleich $1 / 2$ für $\omega_{S} \tau_{\mathrm{c}} \ll 1$, oder: zwischen den Radikalionen und Lösungsmittelprotonen bestehen aus-

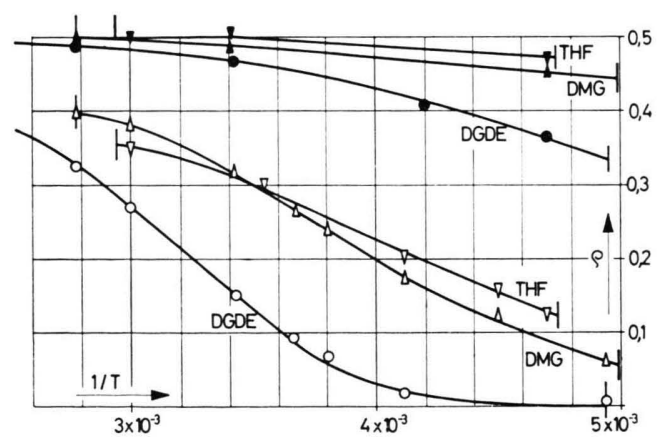

Abb. 6. Meßergebnisse für den Kopplungsparameter des Overhauser-Effehis. Der Flüssigkeitsbereich der Substanzen ist durch senkrechte Linien gekennzeichnet, welche die Kurven abschließen. Dunkle Punkte: $\omega_{\mathrm{S}}=2,74 \cdot 10^{8} \mathrm{sec}^{-1}$, helle Punkte: $\omega_{\mathrm{s}}=1,89 \cdot 10^{10} \mathrm{sec}^{-1}$. schließlich Dipolkopplungen. Ein skalarer Beitrag hätte wegen $\varrho=-1$ die Werte verkleinern müssen.

Die Endergebnisse für die Temperatur- und Frequenzabhängigkeit des Kopplungsparameters $\varrho$ sind in Abb. 6 dargestellt worden. Wie zu erwarten war, haben verschieden konzentrierte Proben einer Substanz zu denselben $\varrho$-Werten geführt. Lösungen mit höherer Radikalkonzentration liefern nur durch gröBere $f$-Werte höhere $P / P_{0}$. An Hand von Abb. 6 konnte geprüft werden, inwieweit die Ergebnisse mit möglichen berechneten Abhängigkeiten (17) oder (20) in Einklang zu bringen sind. Dabei darf zwar die Frequenzabhängigkeit der Spektraldichtefunktionen (16) und (18), nicht aber die $\tau_{\mathrm{c}^{-}}$-Abhängigkeit benutzt werden, da $\tau_{\text {c }}(T)$ a priori nicht bekannt ist. Aus dem schrittweisen Vergleich von Meßwerten $\varrho$ bei gleicher Temperatur, aber verschiedener Frequenz mit möglichen Funktionsverläufen folgt Abb. 7: die Abhängigkeit (17) mit den aus Abb. 2 ermittelten Spektralfunktionswerten steht mit allen Meßergebnissen in Einklang. Mit (20) (gestrichelte Kurve) läßt sich auch bei Parallelverschiebung ( $\tau_{\mathrm{c}}$ ist zunächst unbekannt) keine Übereinstimmung herstellen.

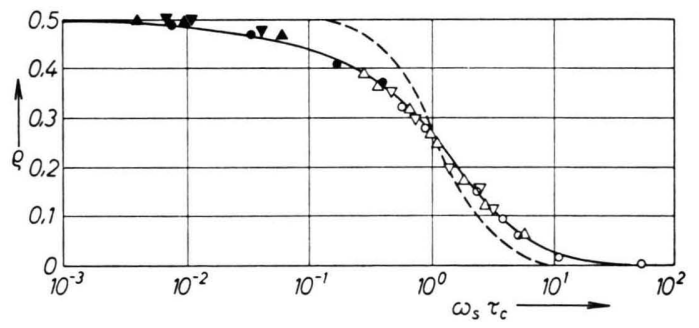

Abb. 7. Der Kopplungsparameter als Funktion von $\omega_{\mathrm{S}} \tau_{\mathrm{c}}$. Durchgezogene Linie: theoretischer Verlauf bei translatorischer Diffusion, gestrichelte Linie bei rotatorischer Diffusion.

\begin{tabular}{|c|c|c|c|}
\hline$\omega_{\mathrm{s}}$ & DGDE & DMG & THF \\
\hline $1,89 \cdot 10^{10} \mathrm{sec}^{-1}$ & $\bigcirc$ & $\triangle$ & $\nabla$ \\
$2,74 \cdot 10^{8} \mathrm{sec}^{-1}$ & $\bullet$ & $\Delta$ & $\nabla$ \\
\hline
\end{tabular}

Aus dem Vergleich von $\varrho(T)$, Abb. 6 und $\varrho\left(\omega_{S} \tau_{\mathrm{c}}\right)$, Abb. 7 ergibt sich die Temperaturabhängigkeit der Korrelationszeit. Ihr in Abb. 8 dargestellter Verlauf folgt einem Gesetz

$$
\tau_{\mathrm{c}}=\tau_{\mathrm{c} 0} \exp \left\{V_{\mathrm{c}} / R T\right\}
$$

mit den in Tab. 1 angegebenen Werten. Dieses Ergebnis kann als unabhängige Bestätigung für den Schluß auf translatorische Diffusion angesehen werden. Auf Grund einer molekular-physikalischen In- 


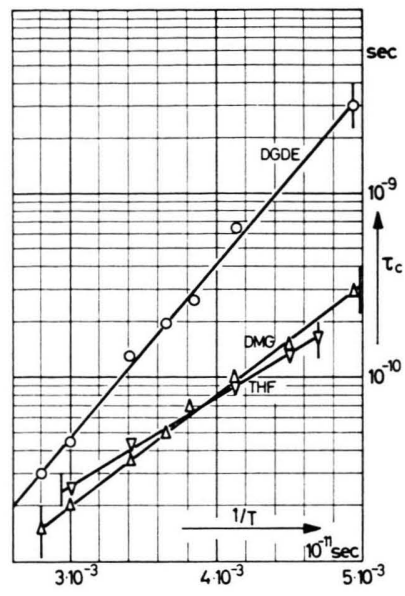

Abb. 8. Korrelationszeiten der translatorischen Molekülbewegung in Abhängigkeit von der Temperatur.

\begin{tabular}{|l|c|c|r|}
\hline & $\tau_{\mathrm{c} 0} / \mathrm{sec}$ & $\begin{array}{l}V_{\mathrm{c}} / \mathrm{kcal} \\
\cdot \mathrm{Mol}^{-1}\end{array}$ & $\tau_{\mathrm{c}}\left(20^{\circ} \mathrm{C}\right) / \mathrm{sec}$ \\
\hline THF & $1,0 \cdot 10^{-12}$ & 2,15 & $4,0 \cdot 10^{-11}$ \\
DMG & $3,5 \cdot 10^{-13}$ & 2,75 & $3,7 \cdot 10^{-11}$ \\
DGDE & $6,5 \cdot 10^{-14}$ & 4,40 & $11,5 \cdot 10^{-11}$ \\
\hline
\end{tabular}

Tab. l. Korrelationszeiten und Aktivierungsenergien für die translatorische Diffusionsbewegung.

terpretation läßt sich die Bewegung der Flüssigkeitsmoleküle durch statistisch in kleinen Bereichen stattfindende Platzwechselvorgänge erklären ${ }^{22}$, woraus sich eine Beziehung von der Art (21) herleiten läßt. Man könnte also eine exponentielle Temperaturabhängigkeit ansetzen, um daraus mittels der Meßwerte von Abb. 6 den Verlauf von Abb. 7 zu gewinnen, wozu dann nur eine Meßfrequenz genügte. Der hier gewählte Weg zeichnet sich jedoch durch größere Allgemeinheit aus.

Quantitative Untersuchungen des OverhauserEffektes haben also zu der Aussage geführt, daß in den untersuchten Radikallösungen Elektronen- und Kernspins nur durch magnetische Dipolwechselwirkungen gekoppelt sind und daß als Bewegungsmechanismus eine translatorische Diffusion angenommen werden kann, die der Gl. (12) gehorcht. Man scheint - wie auch eigene frühere Messungen der Kern- und Elektronenspinrelaxation ${ }^{11,12}$ bestätigen - ganz allgemein in Lösungen freier Radikale eine translatorische Diffusion als Mechanismus für

22 S. Glasstone, K. Laidler u. H. Eyring, Theory of Rate Processes, McGraw-Hill, London 1941.

23 W. Müller-W die Modulation der Wechselwirkungen zwischen den von verschiedenen Molekülen getragenen Spins annehmen zu dürfen. Im Gegensatz zu wäßrigen Lösungen von Ionen der Übergangselemente bilden sich also keine Solvathüllen um die Ionen herum. Der Abfall des Overhauser-Effektes und die maßgeblichen Flüssigkeitsbewegungen können in diesen Stoffen durch eine einzige Korrelationszeit beschrieben werden.

Interessant ist ein Vergleich der Korrelationszeiten von Abb. 8 mit den makroskopischen Viskositäten. Entsprechende Untersuchungen in den gleichen Substanzen haben ergeben, daß diese keinem so einfachen Gesetz wie (21) folgen und daß im allgemeinen nicht die gleichen Aktivierungsenergien angesetzt werden dürfen. Die Viskosität von DGDE, welche die größte Änderung mit der Temperatur zeigt, läßt sich durch zwei Exponentialansätze beschreiben, wobei bei tieferen Temperaturen die gleiche Aktivierungsenergie wie für die translatorische Diffusion maßgeblich ist, für hohe Temperaturen aber eine etwa nur halb so große. Allgemein läßt sich feststellen, daß zur Viskosität nicht unbedingt die gleichen Mechanismen beitragen und daher eine Darstellung der Korrelationszeiten der Molekularbewegungen durch die Viskosität nicht sinnvoll ist.

Die in verschiedenen Flüssigkeiten bei der gleichen Temperatur verschieden großen Kopplungsparameter $\varrho$ sind für alle Anwendungen des OverHauser-Effekts von Bedeutung. Durch den Abfall von $\varrho$ mit $\omega \tau_{\mathrm{c}}$ ist für jede Frequenz und Temperatur eine prinzipielle Grenze für die Größe der erreichbaren Kernpolarisation gegeben. Wenn man sich die Schlußfolgerungen der vorliegenden Untersuchungen $\mathrm{zu}$ eigen macht und für Lösungen freier Radikale eine Abhängigkeit (17) voraussetzt und diese auch

\begin{tabular}{|c|c|c|}
\hline Lösungsmittel & $\tau_{\mathrm{c}}\left(20^{\circ}\right) / \mathrm{sec}$ & $\begin{array}{l}\text { Grenzfrequenz } \\
\text { bei } 20^{\circ} \mathrm{C} / \mathrm{GHz}\end{array}$ \\
\hline THF & $4,0 \cdot 10^{-11}$ & 4,5 \\
\hline DMG & $3,7 \cdot 10^{-11}$ & 5,0 \\
\hline DGDE & $11,5 \cdot 10^{-11}$ & 1,5 \\
\hline Wasser 23 & $0,6 \cdot 10^{-11}$ & 32,7 \\
\hline Benzol $^{23}$ & $3,9 \cdot 10^{-11}$ & 4,6 \\
\hline Aceton 24 & $1,3 \cdot 10^{-11}$ & 13,8 \\
\hline Äther 24 & $1,0 \cdot 10^{-11}$ & 18,0 \\
\hline
\end{tabular}

Tab. 2. ESR-Grenzfrequenzen des Overhauser-Effektes in Lösungen freier Radikale.

24 Die $\tau_{\mathrm{c}}$-Werte wurden aus unveröffentlichten Kernrelaxationsmessungen abgeschätzt. 
nach höheren Frequenzen extrapoliert, so ergeben sich die in Tab. 2 zusammengestellten Grenzfrequenzen, bei denen der Overhauser-Effekt bereits von seinem Maximalwert $\left(\omega_{S} \tau_{\mathrm{c}} \ll 1\right)$ auf die Hälfte abgefallen ist.

Das spezielle Radikal geht in erster Näherung nicht in die Grenzfrequenz ein. Diese läßt sich durch Temperaturerhöhung nach höheren Frequenzen hin verschieben. Ist die Elektronenresonanzfrequenz noch um einen Faktor 10 größer, so ist $\varrho$ auf etwa $5 \%$ seines ursprünglichen Wertes abgefallen. Bei noch höheren Frequenzen oder tiefen Temperaturen verschwindet die Overhauser-Polarisation. Stattdessen läßt sich - wie in einer anderen Arbeit im einzelnen gezeigt worden ist ${ }^{25}$ - in den gleichen Stoffen eine Kernpolarisation durch Festkörpereffekt erzeugen.

Es ist vorgesehen, ähnliche Untersuchungen wie die in diesem Abschnitt diskutierten auch für kompliziertere Flüssigkeitssysteme durchzuführen. Statt der bisherigen zwei sollen dabei weitere Meßfrequenzen benutzt werden.

\section{Protonen- und Elektronen-Spin-Gitter- Relaxation}

Da die gleichen Mechanismen, die die Erzeugung eines Overhauser-Effektes ermöglichen, auch maßgeblich zur Relaxation beitragen, ist eine Untersuchung der Protonen- und Elektronenrelaxationszeiten in den gleichen Substanzen von besonderem Interesse. Die Zusammenhänge werden deutlich, wenn der Informationsgehalt solcher Messungen mit dem des letzten Abschnitts verglichen wird. Die Parameter der BRownschen Molekularbewegung bestimmen die Zeitabhängigkeit der Kopplung zwischen Protonen- und Elektronenspins und der zwischen den Elektronenspins untereinander. Dadurch besteht ein enger Zusammenhang zwischen dem OverhauserEffekt, dem durch Wechselwirkung mit den Elektronen erzeugten Protonenrelaxationsanteil und dem Dipolanteil der Elektronenspinrelaxation. Die entsprechenden Spin-Gitter-Relaxationszeiten $T_{1}^{\prime}$ und $T_{1 \mathrm{e}}$ wurden für beide Meßfrequenzen in Abhängigkeit von der Temperatur gemessen. Abb. 9 zeigt die Ergebnisse. Da die Konzentrationsabhängigkeit der Radikallösungen keine über frühere Untersuchun-

25 K.D. Kramer u. W. Müller-Warmuth, Z. Naturforschg. 18 a, 1129 [1963]. gen ${ }^{11,12}$ hinausgehenden Informationen liefert, sind der übersichtlicheren Darstellung wegen jeweils nur Meßkurven für eine Radikalkonzentration eingetragen.

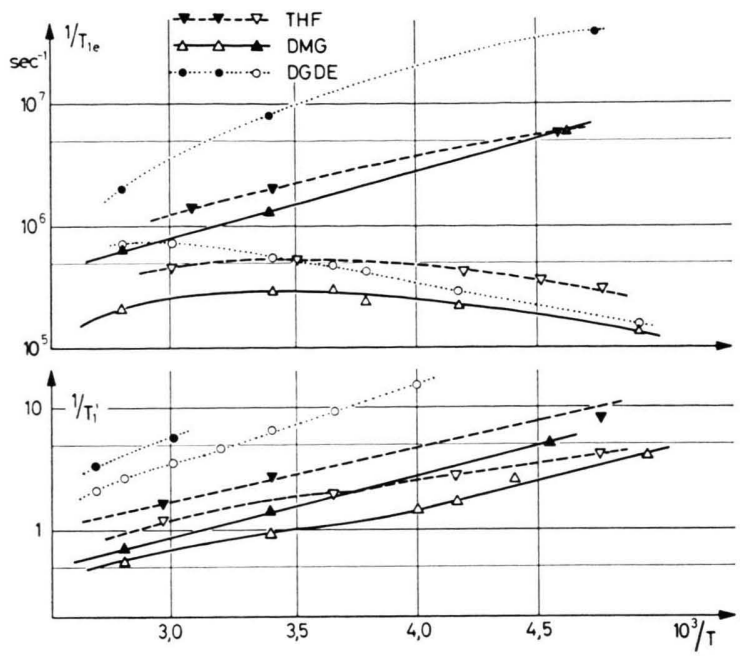

Abb. 9. Meßergebnisse für $T_{1}{ }^{\prime}$ und $T_{1 \mathrm{e}}$ in Abhängigkeit von der Temperatur. Dunkle Punkte: $\omega_{I}=4,08 \cdot 10^{5} \mathrm{sec}^{-1}, \omega_{S}$ $=2,74 \cdot 10^{8} \mathrm{sec}^{-1}$, helle Punkte: $\omega_{I}=2,85 \cdot 10^{7} \mathrm{sec}^{-1}, \omega_{S}$ $=1,89 \cdot 10^{10} \mathrm{sec}^{-1}$. Radikalkonzentrationen: THF $1,0 \cdot 10^{-2}$. $m$., DMG $5,5 \cdot 10^{-3}-m$. und DGDE $2,0 \cdot 10^{-2}-m$.

Zur Messung der Protonenrelaxationszeiten diente bei tiefen Meßfrequenzen eine Kombination der erzwungenen transversalen Präzession mit einer „schnellen“ Modulationsnachweistechnik ${ }^{19}$. Bei der höheren Resonanzfrequenz wurde der makroskopische Magnetisierungsvektor durch einen $180^{\circ}$-Impuls herumgedreht und der Wiederaufbau der Gleichgewichtspolarisation mit normaler Nachweistechnik beobachtet. Zur Messung der Elektronenspinrelaxationszeiten wurden der Vergrößerungsfaktor der dynamischen Polarisation im Maximum der Resonanz für ein bekanntes, sehr kleines $H_{1}$ und die Linienstruktur bestimmt. Wie in Anm. ${ }^{12}$ gezeigt, läßt sich daraus die Spin-Gitter-Relaxationszeit errechnen, wenn zugleich der auf Sättigung extrapolierte Vergrößerungsfaktor bekannt ist. Die Mikrowellenfeldstärke ergibt sich aus den Daten der Apparatur und der gemessenen Leistung. Das Ergebnis ist unabhängig von der aufgelösten oder nicht aufgelösten Hyperfeinstruktur und bezieht sich bei inhomogener Verbreiterung im Mittel auf ein „Spinpaket“ bzw. eine Hyperfeinstrukturlinie.

Die Interpretation von Abb. 9 geht wieder von den in Abschnitt 1 diskutierten Flüssigkeitsmodellen aus. Die Protonenrelaxationszeit $T_{1}{ }^{\prime}$ ergibt sich aus 
$T_{1}$ in $(4 \mathrm{~b})$, wenn man den Anteil der Relaxation des reinen Lösungsmittels $w_{1}^{\prime \prime}$ abzieht. Mit (10) folgt dann für $J_{A}=0$, wenn man $\omega_{S} \gg \omega_{I}$ berück. sichtigt und $J_{j}(\omega) \equiv c_{j} \cdot J(\omega)$ setzt [Definition der $c_{j}$ unter (14)]:

$$
\begin{aligned}
\frac{1}{T_{1}^{\prime}} & =w_{0}+2 w_{1}^{\prime}+w_{2} \\
& =\frac{\pi}{5} \gamma_{S}^{2} \gamma_{I}^{2} \hbar^{2}\left[7 J\left(\omega_{S}\right)+3 J\left(\omega_{I}\right)\right] .
\end{aligned}
$$

Für das Vorliegen einer rein translatorischen Relativbewegung der die Spins tragenden Moleküle soll wieder die Spektraldichte (16) benutzt werden. Wenn man die darin auftretende Integralfunktion mit $f\left(\omega \tau_{\mathrm{c}}\right)$ abgekürzt, ergibt sich aus $(22)$

$$
\frac{1}{T_{1}^{\prime}}=\frac{6 \pi}{5} \gamma_{S}^{2} \gamma_{I}^{2} \hbar^{2} N \frac{\tau_{\mathrm{c}}}{d^{3}} \cdot\left[7 f\left(\omega_{S} \tau_{\mathrm{c}}\right)+3 f\left(\omega_{I} \tau_{\mathrm{c}}\right)\right] \text {. }
$$

Dieser Ausdruck geht für den Grenzwert

$$
f\left(\omega_{S} \tau_{\mathrm{c}}\right)=f\left(\omega_{I} \tau_{\mathrm{c}}\right)=2 / 15
$$

für $\omega_{S} \tau_{\mathrm{c}} \ll 1$ in die bekannte Beziehung

$$
\frac{1}{T_{1}^{\prime}}=\frac{8 \pi}{5} \gamma_{S}^{2} \gamma_{I}^{2} \hbar^{2} N \frac{\tau_{\mathrm{c}}}{d^{3}}
$$

über. Für den allgemeinen Fall ist der Verlauf des von $\tau_{\mathrm{c}}$ und $\omega$ abhängigen Teils von $1 / T_{1}{ }^{\prime}$ nach (23) unter Benutzung der Integralberechnung von Abb. 2 in Abb. 10 wiedergegeben. Die beiden Kurven entsprechen den Arbeitsfrequenzen von $65 \mathrm{kHz}$ und 4,54 MHz. Unter Benutzung von (23) sind die Meß-

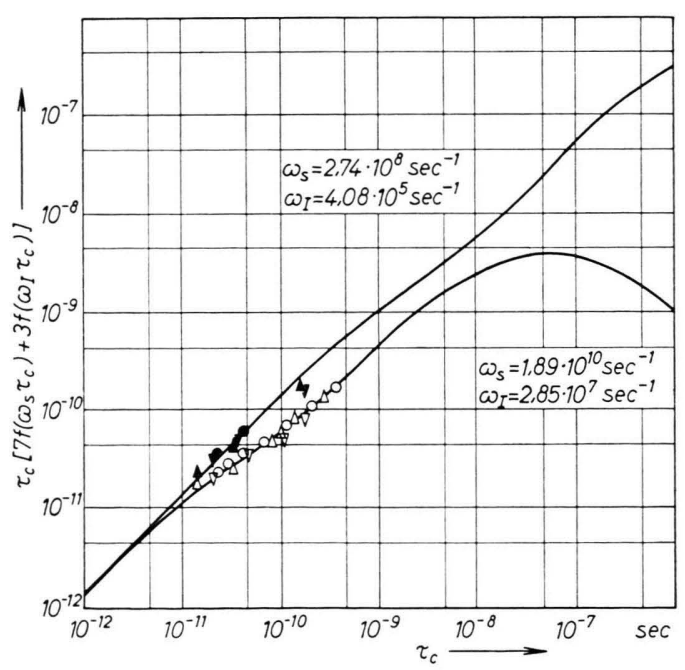

Abb. 10. Verlauf von $\tau_{\mathrm{c}}\left[7 f\left(\omega_{S} \tau_{\mathrm{c}}\right)+3 f\left(\omega_{I} \tau_{\mathrm{c}}\right)\right]$ für die Protonenresonanzfrequenzen $4,54 \mathrm{MHz}$ und $65 \mathrm{kHz}$. Die Meßpunkte für $1 / T_{1}^{\prime}$ von Abb. 9 sind nach (23) möglichst gut an die Kurven angepaßt worden. punkte von Abb. 9 so in diese Darstellung eingetragen, daß sie sich dem theoretischen Kurvenverlauf am besten anpassen. Da die Elektronenspinzahl je $\mathrm{cm}^{3} \quad N$ gemessen ist, geht hierbei als einzige frei wählbare Unbekannte $d$ ein. Die gezeichnete Anpassung entspricht den in Tab. 3 gegebenen Werten für $d$. Für die Umrechnung $\tau_{\mathrm{c}}(T)$ ist hier der aus Messungen des Overhauser-Effektes gewonnene $\mathrm{Zu}$ sammenhang von Abb. 8 benutzt worden. Die Ermittlung von $\tau_{\mathrm{c}}$ aus den Relaxationsmessungen selbst ist in diesem Fall problematisch, da sich die Kurven für die beiden Meßfrequenzen zu wenig unterscheiden.

Für die Berechnung des (bei den vorliegenden Radikalkonzentrationen vorherrschenden, vgl. ${ }^{12}$ ) dipolaren Anteils der Elektronenspinrelaxation aus den Flüssigkeitseigenschaften können ähnliche Betrachtungen wie im Falle der Kernrelaxation angestellt werden. Dabei wird von einer (6) entsprechenden magnetischen Wechselwirkung zwischen zwei (gleichartigen) Elektronenspins ausgegangen. Die Rechnung führt zu einer Übergangswahrscheinlichkeit je Zeiteinheit

$$
w_{\mathrm{e}}=\frac{9}{16} \gamma_{S}^{4} \hbar^{2}\left[J_{1}\left(\omega_{S}\right)+J_{2}\left(2 \omega_{S}\right)\right]
$$

und im Falle translatorischer Diffusion der Radikalionen in der Lösung zu dem mit (23) vergleichbaren Ausdruck

$$
\frac{1}{T_{1 \mathrm{e}}}=\frac{18 \pi}{5} \gamma_{S}^{4} \hbar^{2} N \frac{\tau_{\mathrm{c}}}{d^{3}}\left[f\left(\omega_{S} \tau_{\mathrm{c}}\right)+4 f\left(2 \omega_{S} \tau_{\mathrm{c}}\right)\right]
$$

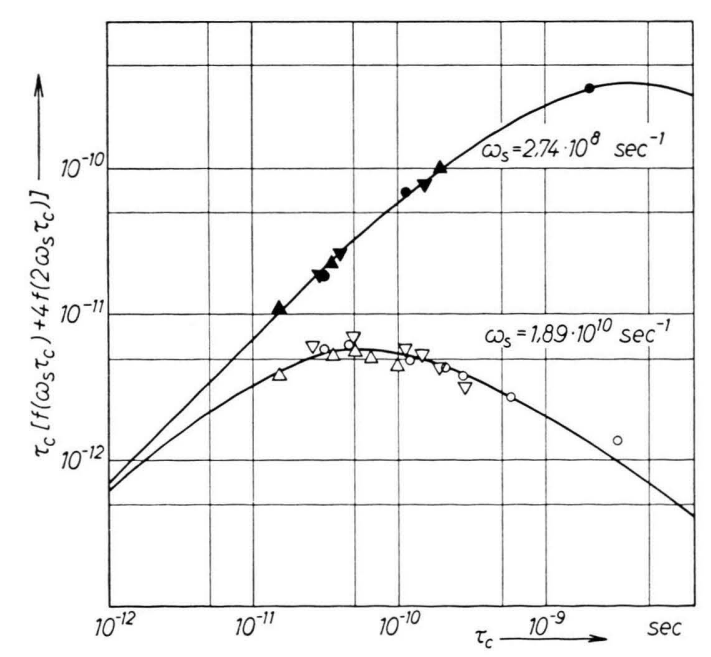

Abb. 11. Verlauf von $\tau_{\mathrm{c}}\left[f\left(\omega_{S} \tau_{\mathrm{c}}\right)+4 f\left(2 \omega_{S} \tau_{\mathrm{c}}\right)\right]$ für die Elektronenresonanzfrequenzen $3,0 \mathrm{GHz}$ und $43,6 \mathrm{MHz}$. Die Meßpunkte für $1 / T_{1 \mathrm{e}}$ von Abb. 9 sind nach (25) möglichst gut an die Kurven angepaßt worden. 
Den Verlauf des von $\tau_{\mathrm{c}}$ abhängigen Anteils zeigt Abb. 11 für beide Frequenzen. Die Meßpunkte von Abb. 9 sind unter Benutzung von Abb. 8 wieder so eingetragen worden, daß sie sich den Kurven möglichst gut anpassen. Die daraus nach (25) resultierenden $d$-Werte enthält Tab.3. Die theoretischen Relaxationsraten besitzen unter den gemachten Annahmen (12) und (16) ein Maximum für $\omega \tau_{\mathrm{c}}$ $=1,04$. Für das Maximum der unteren Kurve $\left(\tau_{\mathrm{c}}=5,5 \cdot 10^{-11} \mathrm{sec}\right)$ beträgt das theoretische Verhältnis der Relaxationsrate bei der tiefen Frequenz zu der bei der höheren 5,3. Das kann als unabhängiges Kriterium für die Deutung der Meßergebnisse von Abb. 7 herangezogen werden. Tatsächlich ist die genannte Bedingung bei den drei Substanzen für die jeweilige Temperatur des Maximums von $1 / T_{1 \mathrm{e}}(3 \mathrm{GHz})$ im Rahmen der Meßgenauigkeit erfüllt. Bei einem anderen Flüssigkeitsmodell ergäbe sich ein anderes Verhältnis; z. B. führen Spektralfunktionen der Form (18) in (24) ebenfalls zu Kurvenverläufen mit einem (schärferen) Maximum. Das Verhältnis der Relaxationsraten beträgt dann aber 2 statt 5,3.

Die Ergebnisse der Relaxationsuntersuchungen lassen sich in Übereinstimmung mit den Messungen des Overhauser-Effektes durch Spektraldichtefunktionen der Art (16) interpretieren. Für eine Bestimmung der Korrelationszeiten erscheint die Methode der $\varrho$-Bestimmung günstiger. Im vorliegenden Fall liegen dafür zudem die Protonenresonanzfrequenzen zu niedrig. Bei den Elektronenspinrelaxationszeiten reicht die Genauigkeit nicht aus. Wegen der Unsicherheit der $H_{1}$-Eichung und der eingehenden Doppelresonanzmessungen ist für die Absolutbestimmung von $T_{1 \mathrm{e}}$ mit einem mittleren Meßfehler von etwa $40 \%$ zu rechnen. Der relative Kurvenverlauf ist dagegen wesentlich genauer.

Wenn man alle erhaltenen Informationen heranzieht, erhält man Aussagen über die Güte von Absolutberechnungen der Relaxationszeiten in ZweiSpin-Systemen. Wenn man alle Messungen verwertet und die vorliegenden Bewegungsmechanismen als geklärt ansieht (hier allein translatorische Diffusion), bleiben als einzige Unbekannte in (23) und (25) die Größen $d$ übrig, die in die Integration $(7 \mathrm{~b})$ und in die Definition der Korrelationszeiten (15) als kleinstmöglicher Abstand zwischen den als starre Kugeln gedachten Wechselwirkungspartnern eingehen. Genau genommen sind die in (17), (23) und (25) auftretenden $\tau_{\mathrm{c}}$ und $d$ nicht ganz die glei- chen, da die Partner einmal von Radikal- und Lösungsmittelmolekül, das andere Mal nur von Radikalmolekülen getragen werden. Diese Unterschiede sind aber sehr klein, weil das Bewegungsverhalten in vorherrschendem Maße von den Lösungsmitteln allein bestimmt wird und weil alle Moleküle vergleichbare Größe besitzen. In Tab. 3 sind die aus den Messungen gewonnenen $d$-Werte in $\AA$ mit berechneten verglichen.

\begin{tabular}{|l|c|c|c|c|}
\hline Lösung & aus $T_{1}^{\prime}$ & aus $T_{1 \mathrm{e}}$ & $\begin{array}{c}\text { aus } \\
\text { Kugel- } \\
\text { packung }\end{array}$ & $\begin{array}{c}\text { aus } \\
\text { STUART- } \\
\text { BrIEGLE- } \\
\text { Kalotten }\end{array}$ \\
\hline THF & 4,8 & 4,6 & 5,6 & 5,0 \\
DMG & 4,7 & 4,6 & 6,0 & 5,0 \\
DGDE & 5,1 & 5,0 & 6,8 & 5,8 \\
\hline
\end{tabular}

Tab. 3. Vergleich der $d$-Werte in $\AA$.

Zur Berechnung von $d$ wurden in Spalte 4 die effektiven Molekülradien aus dem Flüssigkeitsvolumen abgeschätzt. Da man über die Packung in den Flüssigkeiten keine genaue Vorstellung besitzt, diente dabei als Modell eine hexagonal dichteste Kugelpackung der als Kugeln gedachten Moleküle. Wenn $M$ das Molekulargewicht, $L$ die Loschмidт-Zahl und $o$ die Dichte bedeuten, ergibt sich dann

$$
(d / 2)^{3}=M / 5,65 L \varrho .
$$

Ein verfeinertes Modell geht von dem bekannten Bau der Moleküle aus. Man bestimmt die Molekülvolumina aus der Summe der Atomvolumina und bedient sich vorteilhaft der in $1,5 \cdot 10^{8}$-facher Vergrößerung erhältlichen Atomkalottenmodelle von Stuart und Briegleb ${ }^{26}$. Die in Spalte 5 von Tab. 3 aufgeführten Werte haben sich aus diesen Volumina ergeben, wobei die Moleküle durch Kugeln gleichen Volumens ersetzt worden sind.

Die Übereinstimmung ist überraschend gut. Die systematisch zu großen Werte aus der Kugelpackung lassen darauf schließen, daß die wirkliche Packung lockerer erfolgt. Die größten Abweichungen ergeben sich beim DGDE-Modell, dessen langgestreckte Form am wenigsten eine Idealisierung als Kugel zuläßt. Auch die Abschätzungen nach den Stuartschen Modellen scheinen allgemein etwas zu große Werte zu liefern. Zusammenfassend kann jedoch festgestellt werden, daß die ausschließliche Berück-

${ }^{26}$ H. A. Stuart, Die Struktur des freien Moleküls, Verlag Springer, Berlin 1952. 
sichtigung von Volumeneffekten der diffundierenden Moleküle recht wirksam ist. Für die Zwei-Spin-Systeme, in denen die wechselwirkenden Spins zu verschiedenen Molekülen gehören, kann auf diese Weise eine die Experimente relativ genau beschreibende Absolutberechnung der Relaxationszeiten vorgenom- men werden, wenn für die Molekülbewegung ein translatorischer Diffusionsmechanismus ausschlaggebend ist.

Der Deutschen Forschungsgemeinschaft, Bad Godesberg, danken wir für die Unterstützung dieser Arbeiten durch Sachbeihilfen.

\title{
Regelmäßige Relaxationsschwingungen in kurzen Rubin-Lasern vom ebenen Fabry-Perot-Typ*
}

\author{
Von Dieter Röss

\begin{abstract}
Zentral-Laboratorium der Siemens \& Halske AG, München
\end{abstract} \\ (Z. Naturforschg. 19 a, 387-391 [1964] ; eingegangen am 21. November 1963)
}

\begin{abstract}
Das zeitliche Schwingungsverhalten kurzer Rubin-Laser vom ebenen FABrY-Perot-Typ wird wesentlich vereinfacht, wenn das Pumplicht rotationssymmetrisch zur Achse des Lasers eingestrahlt wird. Das konnte in einer neuartigen Pumpanordnung mit hohem Wirkungsgrad erreicht werden, bei der Laser und Lichtquelle in der Achse eines Rotationsellipsoid-Spiegels exfokal angeordnet sind. Die Relaxationsimpulse folgen dann auch bei hoher Pumpenergie nahezu regelmäßig aufeinander.

Bei sehr niedriger Pumpleistung oder bei hoher Auskopplung aus dem Resonator treten gedämpfte periodische Relaxationsschwingungen mit kontinuierlicher Emission auf.

Die bei hoher Quantendichte im Resonator beobachtete Entdämpfung der Relaxationsschwingungen führen wir auf Effekte zurück, die in der Theorie bisher nicht berücksichtigt worden sind. Für die Regelmäßigkeit der Relaxationsschwingungen ist eine ungestörte Kopplung zwischen den Moden wichtig.

An einem Laser hoher Modenselektion mit Etalon-Reflektor wurde eine zeitliche Aufeinanderfolge isolierter Relaxationsschwingungen verschiedener Moden beobachtet.
\end{abstract}

\section{Optische Pumpanordnung mit rotations- symmetrischer Beleuchtung}

Für die Beleuchtung optisch gepumpter Laser werden vorwiegend zwei Pumpsysteme verwendet: Einmal eine spiralige Blitzlampe, in deren Innenraum der Laser eingebracht wird ${ }^{1,2}$; zum anderen elliptische Zylinderspiegel, die eine in einer Brennlinie angeordnete Stabblitzlampe auf den in der anderen Brennlinie angeordneten Laser abbilden ${ }^{3}$. Neben ciner unvollständigen Ausnutzung der Lichtquelle haben beide Pumpsysteme weitere Nachteile: Der Laser befindet sich in einem von der Lichtquelle erzeugten inhomogenen Magnetfeld, das zu einer Aufspaltung und Verbreiterung der Niveaus führt. Außerdem wird der Laser vom Pumplicht in einer nichtrotationssymmetrischen Verteilung getroffen ${ }^{4}$,

* Auszugsweise vorgetragen auf der Deutschen Physikertagung Hamburg 1963.

1 T. H. Maiman, R. H. Hoskins, I. J. D'Haenens, C. K. Asawa u. V. Evtuhov, Phys. Rev. 123, 1151 [1961].

2 R. J. Collins, D. F. Nelson, A. L. Schawlow, W. Bond, C. G. B. Garret u. W. Kaiser, Phys. Rev. Letters 5, 303 [1960]. was zu einer unsymmetrischen Absorption des Pumplichts im Querschnitt führt. Wie wir früher gezeigt haben ${ }^{5}$, kann sich daraus eine Verbiegung des Laserstabes und eine Verstimmung des Resonators während des Pumpimpulses ergeben. Wir beobachteten in einer solchen Anordnung z. B., daß die Schwelleistung keine Konstante ist, sondern von der Pumpenergie abhängt, und daß die Laserschwingung bei höherer Pumpenergie eher aufhören kann als bei niedrigerer Pumpenergie.

Wir haben ein Beleuchtungssystem mit hohem Wirkungsgrad entwickelt, in dem das Magnetfeld der Lichtquelle am Ort des Lasers vernachlässigbar klein ist und in dem das Pumplicht den Laser in genauer Rotationssymmetrie durchsetzt. Das System, über das wir an anderer Stelle ausführlich berichtet haben ${ }^{6}$, ist in Abb. 1 in einer praktischen Ausführungsform dargestellt. Die Lichtquelle befindet sich in einem Rotationsellipsoid-

\footnotetext{
3 M. Ciftan, C. F. Luck, C. G. Shafer u. H. Statz, Proc. I. R. E. 49, 960 [1961].

4 D. Röss, Frequenz 16, 423 [1962].

5 D. Röss, Frequenz 17, 61 [1963].

${ }_{6}$ D. Röss, Appl. Opt. 3, 259 [1964].
} 